\title{
Caracterização do atendimento de uma Unidade de Hemoterapia
}

\author{
Characterization of the assistance of a Hematology Unit \\ Caracterización de la asistencia de una Unidad de Hematología
}

\section{Rodrigo Guimarães dos Santos Almeida', Alessandra Mazzo", Isabel Amélia Costa Mendes", Maria Auxiliadora Trevizan", Simone de Godoy"}

' Unidade Hemoterápica de Batatais. Batatais-SP. Universidade de São Paulo, Faculdade de Medicina de Ribeirão Preto, Mestrado em Saúde da Comunidade (Mestrando). Ribeirão Preto-SP, Brasil.

" Universidade de São Paulo, Escola de Enfermagem de Ribeirão Preto, Departamento de Enfermagem Geral e Especializada. Ribeirão Preto-SP, Brasil.

Submissão: 24-09-2010Ａprovação: 27-01-2012

\begin{abstract}
RESUMO
O estudo objetivou descrever as características de atendimento de uma Unidade de Hemoterapia do interior do estado de São Paulo, no ano de 2009. Seguidos os preceitos éticos, foi realizado levantamento junto ao banco de dados da Unidade. No período observado a Unidade demonstra maior índice de doadores de repetição, com baixo índice de reações adversas e $100 \%$ de satisfação dos doadores. No serviço hospitalar cliente da Unidade, a clínica médica e a unidade de terapia intensiva foram os setores com maior consumo de transfusões. A Unidade descrita é autossuficiente no gerenciamento dos seus recursos. O presente estudo aponta para a necessidade de se explorar as dimensões dos papéis profissionais em hemoterapia, as possibilidades de captação de novos doadores, os motivos que levam os pacientes a doação de sangue, características, perfil epidemiológico e satisfação dos receptores.

Descritores: Enfermagem; Assistência à saúde; Doadores de Sangue; Garantia da Qualidade dos Cuidados de Saúde.
\end{abstract}

\begin{abstract}
This study aimed to describe the characteristics of service in a Hemotherapy Unit in the interior of the state of São Paulo in 2009. All ethical guidelines were followed and a survey was carried out in the database of the Unit. During the observed period, the Unit shows greater rate of repetition donors, with low levels of adverse reactions and $100 \%$ of donors' satisfaction. The units of clinical medicine and intensive therapy were the sectors with highest consumption of transfusions at the hospital service. The described unit is self-sufficient at managing its resources. The present study shows the need to explore the dimensions of the professional roles in hemotherapy, the possibilities of finding new donors, the reasons that lead patients to donate blood, characteristics, epidemiological profile and satisfaction of receptors.
\end{abstract}

Key words: Nursing; Health Care; Blood Donors; Quality Assurance; Delivery of Health Care.

\section{RESUMEN}

El objetivo del estudio es describir las características de atendimiento de una Unidad de Hemoterapia del interior del estado de São Paulo en el año de 2009. Fueron seguidos los preceptos éticos y realizado sondeo en la base de datos de la Unidad. En el periodo observado la Unidad muestra mayor índice de donadores de repetición, con bajo índice de reacciones adversas y el $100 \%$ de satisfacción de los donadores. Los sectores de clínica médica y la unidad de terapia intensiva fueron los de mayor consumo de transfusiones en el servicio hospitalario cliente de la Unidad. La Unidad descripta es auto-suficiente en el gerenciamiento de sus recursos. El presente estudio apunta a la necesidad de explorar las dimensiones de los papeles profesionales en hemoterapia, las posibilidades de captación de nuevos donadores, los motivos que llevan los pacientes a la donación de sangre, y las características, perfil epidemiológico y satisfacción de los receptores.

Descriptores: Indicadores; Enfermería; Atención de Salud; Donadores de Sangre; Garantía de la Calidad de Atención de Salud. 


\section{INTRODUÇÃO}

A hemoterapia é uma importante área de estudos, uma vez que compreende o uso de um grande número de recursos materiais e humanos. Visa à produção de serviços, produtos e o atendimento de clientes (usuários, associados, contribuintes e consumidores). É composta pelo setor público (hemocentros, núcleos e unidades) e privado (serviços de hemoterapia e bancos de sangue $)^{(1)}$.

Como um serviço da saúde, deve buscar a qualidade pela identificação de falhas nas rotinas e procedimentos e a condução de melhoria dos processos e resultados. Necessita estabelecer, como meta, a conformidade com as exigências dos órgãos reguladores e a satisfação dos clientes atendidos. Em serviços de hemoterapia, compete à Vigilância Sanitária a avaliação dos serviços prestados ${ }^{(2)}$.

Desde 1980, com a criação do Programa Nacional de Sangue e Hemoderivados - PRO-SANGUE, muitos avanços têm sido alcançados na hemoterapia no Brasil(3). Ainda assim, é constante nos meios de comunicação a divulgação da falta de sangue para transfusões, o que torna esse um dos problemas atualmente destacados dentre as múltiplas necessidades da saúde pública ${ }^{(4)}$. Existe a necessidade do aumento no número de doadores para que os estoques e as demandas de sangue para transfusões sejam atendidos ${ }^{(5)}$.

Entretanto, para que o número de doações de sangue seja expressivo, é necessário que se estabeleça, entre instituições, profissionais, doadores e métodos utilizados, uma relação de confiança e segurança na perícia e na intenção dos profissionais $^{(1)}$.

A hemoterapia é um dos diversos segmentos da saúde onde os profissionais de enfermagem exercem sua prática. Nesse contexto, o enfermeiro desempenha um importante papel, seja no atendimento a doadores e/ou a receptores, na busca constante em disponibilizar serviços e produtos de qualidade, na produção de hemocomponentes, no atendimento assistencial e no desenvolvimento do ensino e da pesquisa no setor ${ }^{(6)}$.

A Resolução no 306/2006, do Conselho Federal de Enfermagem, determina, em seu artigo $1^{\circ}$, como competências e atribuições do enfermeiro em Hemoterapia, entre outras, que ele possa assistir de maneira integral os doadores, receptores e suas famílias, promovendo ações preventivas, educativas e curativas entre receptores, familiares e doadores; triagem clínica para avaliação de doadores e receptores; além das ações relacionadas à supervisão e controle da equipe de enfermagem ${ }^{(7)}$.

Para o alcance dessas metas, é imprescindível que enfermeiro de Hemoterapia busque o desenvolvimento de ações que possam promover a qualidade e garantir a produção dos serviços realizados e prestados. Nesse sentido, dentre as várias atividades propostas é sua função reconhecer a população de doadores onde desenvolve suas atividades, os principais motivos de recusa para que possa assegurar maior confiabilidade ao sangue coletado, verificar a produção de hemocomponentes a partir das doações realizadas; conscientizar a população de doadores quanto à importância de realizar o cadastro de possíveis doadores de medula óssea; entre outras ${ }^{(1,8)}$. Por se tratar de um trabalho árduo e extenso, e no intuito de que seja efetivamente realizado, o enfermeiro de hemoterapia precisa reconhecer as características do local onde está inserido, para que possa identificar e equacionar os problemas existentes e traçar metas de trabalho.

Assim, o objetivo do presente estudo é descrever as características de atendimento de uma Unidade de Hemoterapia do interior do estado de São Paulo no ano de 2009.

\section{MATERIAL E MÉTODOS}

Trata-se de estudo descritivo exploratório, aprovado pelo Comitê de Ética em Pesquisa da Escola de Enfermagem de Ribeirão Preto da Universidade de São Paulo - USP, sob parecer $1146 / 2010$.

Os dados foram coletados junto ao Banco de Dados (Sistema de Banco de Sangue - SBS) de uma Unidade de Hemoterapia localizada no interior do estado de São Paulo. Segundo dados da Fundação Sistema Estadual de Análise de Dados - SEADE, em 2009, a população estimada na cidade era de 56.271 habitantes, sendo 34.867 com faixa etária entre 20 e 64 anos de idade ${ }^{(9)}$.

A Unidade de Hemoterapia desse estudo faz parte do complexo de agências, unidades e núcleos transfusionais que compreendem um Hemocentro Regional e está localizada dentro da única instituição de nível hospitalar terciário da cidade onde se localiza. Caracteriza-se por realizar atividades de atendimento a doadores, atendimento das necessidades transfusionais da unidade terciária dentro da qual está localizada e de uma unidade hospitalar de pequeno porte localizada numa cidade vizinha. Conta com uma equipe multidisciplinar composta por um médico, um enfermeiro, duas biomédicas e dois agentes de captação de doadores. Ao enfermeiro competem as atividades de pré-triagem, triagem clínica e coleta de sangue.

Para a realização do estudo, foram coletados dados referentes ao período de janeiro a dezembro de 2009, quanto à caracterização das doações realizadas na unidade, índice de fracionamento (bolsas duplas e triplas), número de coletas enviadas ao Registro Nacional de Doadores de Medula Óssea (REDOME), principais causas de recusa na triagem clínica, tempo de permanência do doador na unidade, índice de satisfação do cliente/doador com a unidade, número de tipagens sanguíneas, pesquisa de anticorpos irregulares e provas de compatibilidade. Os dados foram compilados mensalmente, transportados para o programa Excel, analisados e apresentados na forma de relatório discursivo.

\section{RESULTADOS}

Dentre os 2.873 candidatos a doação no ano de 2009, o total de rejeições na triagem clínica foi de $80(2,7 \%)$, sendo a maior média de rejeição a registrada no mês de junho 16 $(8,9 \%)$ e a menor em dezembro (nenhuma rejeição encontrada neste mês). Os motivos que levaram à rejeição na triagem clínica foram o intervalo entre doações em 44 (55,0\%) candidatos e relacionamento sexual de risco em 36 (45,0\%) candidatos.

Conforme a SEADE no ano estudado, a unidade realizou 2777 coletas, o que representa cerca de 4,9\% de doação/ 
habitante/cidade ${ }^{(9)}$. Destas, 1775 (64,0\%) foram realizadas por doadores de repetição (indivíduo que doa sangue pelo menos uma vez a cada 13 meses), 584 (21,0\%) por doadores esporádicos (indivíduos que doam sangue eventualmente com intervalo maior que 13 meses) e 418 (15,0\%) por novos doadores (indivíduo que doa sangue pela primeira vez num serviço de hemoterapia). A distribuição mensal do número de doadores de repetição, novos e esporádicos está representada no Gráfico 1.

O mês em que ocorreu o maior número de coletas foi março $319(11,5 \%)$, e o menor número de coletas foi registrado em junho $178(6,4 \%)$. Do total de bolsas coletadas no ano de $2009,1.505$ (54,2\%) eram bolsas duplas (concentrado de hemácias e plasma) e $1.272(45,8 \%)$ bolsas triplas (concentrado de hemácias, plasma e plaquetas).

A maior média registrada em doações de repetição é observada nos meses de junho 125 (70,2\%), e a menor no mês de março $184(57,6 \%)$, conforme demonstra o Gráfico 1. Dentre os novos doadores da Unidade, a maior média registrada ocorreu em março 64 (20,0\%), e a menor em dezembro 23 $(11,6 \%)$ e de doadores esporádicos a maior média registrada foi em janeiro 68 (25,8\%) e a menor em agosto 35 (14,7\%).

Em 2009 foram registradas 34 reações adversas à doação de sangue, o que representa uma média mensal em torno de

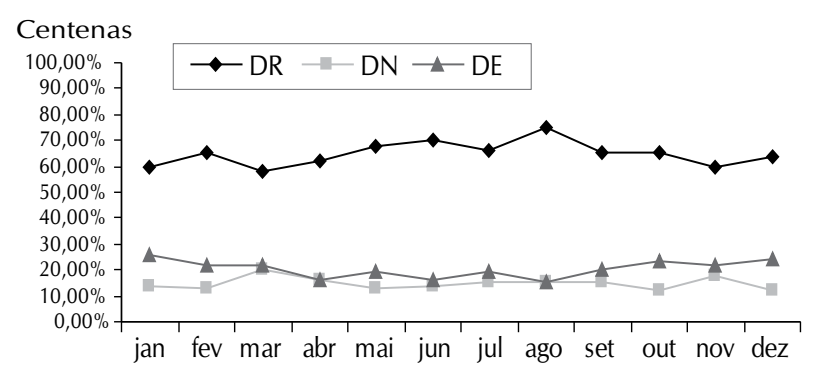

\section{Gráfico 1 - Distribuição do número de doadores de repetição (DR), novos (DN) e esporádicos (DE) da unidade. Batatais-SP, 2009.}

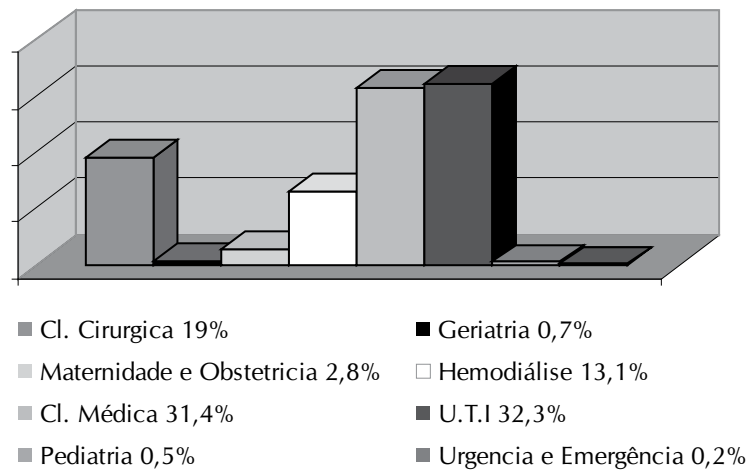

Gráfico 2 - Distribuição do número de hemocomponentes dispensados pela Unidade de Hemoterapia à instituição terciária, por unidade. Batatais-SP, 2009.
1,20\% das doações. A maior incidência registrada de reações adversas foi em janeiro com $7(2,7 \%)$ e a menor em junho (nenhuma reação adversa foi registrada nesse mês). Dentre essas, $30(88,2 \%)$ foram caracterizadas como reações leves, 3 $(8,8 \%)$ como moderadas e 1 ( $3 \%$ ) como grave. O doador que apresentou a reação grave foi atendido na própria unidade.

Durante o período do estudo foram dispensadas pela unidade 940 bolsas de hemocomponentes. Destas, $913(97,1 \%)$ foram destinadas à unidade de atendimento terciário onde a unidade se localiza, sendo 781 concentrados de hemácias e 132 plasmas frescos congelados, $286(31,4 \%)$ foram destinadas a unidade de clínica médica e 295 (32,3\%) a unidade de terapia intensiva. As outras 27 bolsas (22 concentrados de hemácias e 5 plasmas frescos congelados) foram encaminhadas para a unidade situada na cidade vizinha.

Foram atendidos pela unidade 387 clientes/receptores o que corresponde a 387 tipagens sanguíneas e pesquisa de anticorpos irregulares (PAI) e, realizadas 878 provas de compatibilidade sanguínea, das quais 803 bolsas foram transfundidas. As unidades hospitalares onde as transfusões foram realizadas estão identificadas no Gráfico 2.

A média de tempo de permanência do doador na unidade foi de 52 minutos e 27 segundos, sendo o maior tempo de permanência registrado no mês de janeiro (01:20 h) e o menor em abril (00:34 h).

A avaliação da satisfação dos clientes/doadores é realizada no serviço através de um questionário com perguntas estruturadas, entregues aleatoriamente, a cerca de $25 \%$ dos doadores atendidos. Dos 694 doadores que responderam o instrumento, todos $(100 \%)$ indicaram satisfação com a unidade.

\section{DISCUSSÃO}

O número de doações de sangue em um país deve representar de 3 a $5 \%$ da sua população para atender sua demanda transfusional ${ }^{(10)}$. No serviço estudado, evidencia-se que a oferta de doadores atende sua demanda transfusional e o torna autossuficiente. Estes dados divergem da média nacional, segundo os dados do Minstério da Saúde, onde em 2006, houve 3.065 .352 doações realizadas, o que corresponde a 1,77\% da população $^{(11)}$.

Caracteriza-se como doador de sangue ou componentes o indivíduo com idade mínima de 18 anos completos, e máxima de 65 anos 11 meses e 29 dias; com peso acima de $50 \mathrm{Kg}$ e que tenha boa saúde ${ }^{(12)}$.

O tipo de doação e o tipo de doador de sangue são classificados segundo os critérios adotados pelo Ministério da Saúde do Brasil e Sociedade Brasileira de Hematologia e Hemoterapia. Denomina-se doação espontânea, aquela realizada sem qualquer forma de benefício para o doador e que compreenda a doação de uma unidade de sangue ou de um de seus componentes; doação de reposição é a realizada através de recrutamento do doador com a finalidade de repor uma unidade de sangue ou de um dos componentes utilizados por um determinado paciente; doação autóloga é a doação de sangue ou de um de seus componentes para uso do próprio doador; doação específica, a doação de uma unidade de sangue ou de 
um de seus componentes de um doador específico para um receptor específico e doação por aférese, onde se é possível retirar apenas uma das células do sangue total ${ }^{(11,12)}$.

Segundo a Organização Pan-Americana de Saúde (OPAS) e a Organização Mundial de Saúde (OMS) dentre as metas preconizadas pelo Ministério da Saúde existe a necessidade do aumento do número de doadores espontâneos no Brasil, uma vez que no país predomina a prática da doação de reposição. As doações de reposição, ou com qualquer tipo de interesse ou troca, diminuem a qualidade do sangue captado. As doações espontâneas e de repetição são mais seguras e recomenda-se que sejam realizadas por cerca de $60 \%$ dos doadores, demonstrando que no serviço analisado há uma maior confiabilidade do sangue coletado. Tal dado torna-se relevante uma vez que o emprego de hemoderivados não está isento de riscos. Estima-se que nos Estados Unidos da América (EUA) sejam realizadas cerca de 22 milhões de transfusões sangüíneas por ano, com uma taxa de complicações clínicas de grau variável que chega a 20\%, o que leva a implicações administrativas, logísticas e econômicas às quais o enfermeiro deve estar atento. No entanto, a hemoterapia, no Brasil e no mundo de uma forma geral tem se caracterizado pelo desenvolvimento e adoção de novas tecnologias com o objetivo de minimizar os riscos transfusionais, especialmente quanto à disseminação de agentes infectocontagiosos ${ }^{(13-15)}$.

Para a obtenção de segurança dos produtos sanguíneos utilizados, devem-se seguir rígidos parâmetros de qualidade, que envolvem um conjunto de medidas quantitativas e qualitativas, que minimizam riscos e permitem a formação de estoques de hemocomponentes capazes de atender à demanda transfusional ${ }^{(16)}$.

No Brasil, a Portaria n 1.376/93, reforçada pela Resolução no 343 MS/2001, determina que os Serviços de Hemoterapia realizem testes de triagem sorológica para sífilis, doença de Chagas, hepatite B e C, Aids, HTLV e malária. Quanto aos receptores de sangue, é necessário realizar testes imunohematológicos: $\mathrm{ABO} / \mathrm{Rh}$, pesquisa de anticorpos irregulares (PAI) e testes de compatibilidade ${ }^{(17)}$.

Todavia, embora haja o conhecimento do perfil clínico, epidemiológico e sorológico dos doadores de sangue, não se realiza o mesmo com os receptores de sangue, e em especial com aqueles que necessitam de sangue esporadicamente. Estudo pioneiro no país realizou uma avaliação do perfil sorológico pré transfusional de receptores de sangue envolvendo três grupos de receptores (sem passado transfusional, politransfundidos e eventuais) e os resultados revelaram que 37 (43,6\%) foram reagentes aos mesmos testes de triagem sorológica utilizados para doadores ${ }^{(18)}$. Além dessas considerações, dentre as diversas atividades a serem desenvolvidas, avaliadas e atendidas pelos enfermeiros que atuam em hemoterapia, estão as reações adversas que podem ocorrer em gravidade variável durante ou ao final do procedimento de coleta de sangue ${ }^{(19)}$.

Em geral doadores voluntários de sangue não apresentam reações à sangria. Na maioria das vezes, as reações adversas estão relacionadas a doadores ansiosos ou excitados, e caracterizam-se por alteração no padrão respiratório e hiperventilação, o que pode levar em alguns casos, a queda dos níveis de gás carbônico, alcalose e tetania caracterizada por contraturas musculares involuntárias e espasmos. Podem ocorrer, ainda, em caso de reações graves, reações vaso-vagais, indicadas por palidez, sudorese, tremores, calafrios, sensação de tontura, mal estar, náuseas, vômitos, cefaléia, escurecimento da visão, perda de consciência, convulsões e perda do controle esfincteriano com micção e defecação involuntárias ${ }^{(19)}$.

A Unidade avaliada possui baixo índice de reações quando comparada a outros serviços. Para garantir uma melhor eficácia e padronizar o atendimento aos doadores que apresentam algum tipo de reação, o Hemocentro Regional ao qual está vinculada, desenvolveu uma classificação das reações vaso-vagais. Classifica como reação leve, aquela em que o doador apresenta palidez, sudorese, tremores, calafrios, sensação de tontura, mal estar, náuseas, vômitos, cefaléia, escurecimento sem perda de consciência ou com perda revertida instantaneamente; como reações moderadas, aquelas em que o doador apresenta perda da consciência, revertida rapidamente, após algum estímulo, reação em que não ocorre perda da consciência, mas o doador demora a se recuperar, reação com perda rápida da consciência, associada a uma ou duas contraturas musculares, sem liberação de esfíncter; e como reações graves aquelas em que ocorrem perda de consciência prolongada ou associada a contrações, espasmos musculares, convulsões, angina, infarto ou reações que possam ocasionar sequelas ${ }^{(19)}$. Cabe ao enfermeiro de hemoterapia, o envolvimento e o domínio desta padronização, uma vez que dentre as diversas maneiras de qualificação do serviço, a padronização de rotinas resulta em procedimentos e diferentes formas de conduta profissional que transcendem o posicionamento individual das diferentes categorias profissionais, assegurando um cuidado de enfermagem embasado e científico ${ }^{(20)}$.

As semelhanças encontradas nas características das clínicas receptoras e dos hemoderivados utilizados no serviço estudado com as de outros estudos, demonstram que para planejar, gerenciar suas atividades e assegurar quantidade e qualidade dos produros fornecidos, o enfermeiro de hemoterapia necessita conhecer as características dos serviços clientes, como número de leitos das mais diversas clínicas, características dos pacientes, índices que verifiquem sua gravidade, entre outros, de modo que possa liderar a equipe e processos de trabalho ${ }^{(14-15)}$.

Além disso, cumpre destacar que a busca dos serviços pela qualidade prestada e pela satisfação dos doadores de sangue, está positivamente relacionada à intenção de retorno para uma futura doação e faz parte de um processo no avanço do aperfeiçoamento da gestão ${ }^{(6)}$, o que é imprescindível nos dias atuais. Frequentemente os resultados mais utilizados para avaliação da qualidade dos serviços, têm sido a satisfação do cliente, o que pelos dados encontrados, confere ao serviço do estudo destaque nas suas atividades.

\section{CONCLUSÃO}

As Unidades de Hemoterapia são complexas prestadoras de serviços e, embora estando ligadas a unidades centrais como os Hemocentros, devem gerir seu atendimento com segurança, eficácia e qualidade nos serviços prestados.

A Unidade do estudo, embora esteja localizada numa cidade do interior do estado de São Paulo, tem um alto índice de 
produção, dentro do preconizado pela OMS e pode gerir seus recursos, atender de maneira autônoma sua demanda, além de diferenciar-se na satisfação do cliente e na qualidade do sangue captado pela quantidade de doadores de repetição que possui.

A análise dos dados da Unidade e dos clientes (doadores e receptores) oferece ao enfermeiro da Unidade de Hemoterapia, importantes ferramentas administrativas na gestão do seu trabalho e o seu posicionamento perante a equipe, uma vez que vai do discurso ao julgamento dos fatos e, principalmente possibilita de maneira didática e reflexiva o confronto com os dados literários e recomendações de órgãos oficiais.

O presente estudo aponta para a necessidade de se explorar as dimensões dos papéis profissionais, as possibilidades de captação de novos doadores, os motivos que levam os pacientes a doação de sangue, características, perfil epidemiológico e satisfação dos receptores. Enquanto profissional integrante da equipe dessas unidades é papel do enfermeiro o conhecimento de suas atividades e o estabelecimento de metas pessoais e profissionais no intuito de elevar o crescimento pessoal, profissional e principalmente diferenciar o cuidado de enfermagem prestado.

Compete ainda ao enfermeiro de hemoterapia, enquanto profissional da área da saúde, educador e motivador de uma assistência diferenciada, a análise do interesse e disseminação à população sobre as políticas públicas de incentivo a doação de sangue nas suas unidades de trabalho.

\section{REFERÊNCIAS}

1. Silva KFN, Soares S, Iwamoto $\mathrm{HH}$. A prática transfusional e a formação dos profissionais de saúde. Rev Bras Hematol Hemoter. 2009 Dez 18; 31(6): 421-6.

2. D'Innocenzo M, Adami NP, Cunha ICKO. O movimento pela qualidade nos serviços de saúde e enfermagem. Rev Bras Enferm. 2006 Jan-Fev; 59(1): 84-8.

3. Brasil. Ministério da Saúde. Secretaria Executiva. Programa qualidade do sangue: sangue e hemoderivados. Brasília 2000; 54 p.

4. Pereira RSMR, Cartana MH. Projeto Escola - HMOSC: Na busca da qualidade do sangue e da saúde para todos. Rev Ciências Saúde. 2006 Jun-Dez; 25(2): 51-7.

5. Brasil. Ministério da Saúde. Secretaria de Atenção à Saúde. Departamento da Política Nacional de Sangue e Hemoderivados. Caderno de informação: sangue e hemoderivados: rede física, produção, gastos públicos com hemoterapia e consumo de hemoderivados. Brasília 2008; 146 p.

6. Nguyen DD, Devita DA, Hirschler NV, Murphy EL Blood donor satisfaction and intention of future donation.. Transfusion. 2008 Jan; 48(4): 742-8.

7. Conselho Federal de Enfermagem. Resolução 306 do Conselho Federal de Enfermagem - COFEN. Brasília, 2006. [citado em: 19 mar 2010]. Disponível em: http://www.portalcofen.gov.br

8. Sojka BN, Sojka P. The blood donation experience: selfreported motives and obstacles for donating blood. Vox Sanguinis. 2008 Jan; 94(1): 56-63.

9. Fundação Sistema Estadual de Análise de Dados. Bases de dados. São Paulo, 2010. [citado em 15 jun 2010]. Disponível em: http://www.seade.gov.br

10. Spada C, Treitinger A, Souza MA. Prevalência do tabagismo em doadores de sangue da região serrana de Santa Catarina - Brasil. Rev Bras Hematol Hemoter [serial online] 2006 Mar [cited 2010 Sep 21]; 28(1): 19-23. Available from: http:// www.scielo.br/scielo.php?script $=$ sci_arttext \&pid $=$ S151684842006000100006\&lng $=$ en

11. BRASIL. Ministério da saúde. Parâmetros da Organização Mundial de Saúde para doação de sangue no
Brasil. Brasília, 2010. [citado em: 28 mar 2010] Disponível em: http://portal.saude.gov.br/portal/saude/area. cfm?id_area $=124$

12. Agência Nacional de Vigilância Sanitária - Anvisa. Sangue e hemoderivados. Legislação. Brasília, 2001. [citado em 22 jun 2010]. Disponível em: http://www.anvisa.gov.br/ sangue/legis/index.htm

13. Pereira, RSM, Reibnitz, KS, Martini, JG, Nitschke, RG. Doação de sangue: solidariedade mecânica versus solidariedade orgânica. Rev Bras Enferm [serial online] 2010 Apr [cited 2010 Jun 22]; 63(2): 322-327. Available from: http:// www.scielo.br/scielo.php?script $=$ sci arttext\&pid $=$ S0034 $71672010000200024 \& \operatorname{lng}=\mathrm{en}$

14. Volpato SE, Ferreira JS, Ferreira VLPC, Ferreira DC. Transfusão de concentrado de hemácias em Unidade de Terapia Intensiva. Rev Bras Hematol Hemoter 2005 27(3):17982.

15. Regan F, Taylor C. Recent developments. Blood transfusion medicine. BMJ 2002; 323:143-47.

16. Jullien AM, Courouce AM, Richard D, Favre M, Lefrere JJ, Habibi B. Transmission of HIV by blood from seronegative donors. Lancet 1988; 2(8622):1248-9.

17. Agência Nacional de Vigilância Sanitária (Anvisa). Resolução da Diretoria Colegiada RDC 153 de 14 de Junho de 2004. Brasília, 2004. [citado em 20 abr 2010]. Disponível em: http://www.anvisa.gov.br

18. Carrazzone C, Verçosa AFA, LorenaVMB. Avaliação do perfil sorológico pré-transfusional em receptores de sangue do Hospital Universitário Oswaldo Cruz-UPE. Rev Bras Hematol Hemoter 2003; Supl 2:191-256.

19. Crocco A, D'elia D. Adverse reactions during voluntary donation of blood and/or blood components. Transfus Sangue. 2007 Jul; 5(3): 143-52.

20. Guerrero GP, Beccaria LM, Trevizan MA. Procedimento operacional padrão: utilização na assistência de enfermagem em serviços hospitalares. Rev Latino-Am Enfermagem [serial online] 2008 [cited 2009 Jul 13]; 16(6): 966-72. Available from: http://www.scielo.br/scielo.php?script =sci arttext\&pid $=$ S0104-11692008000600005\&lng $=$ pt. 